\title{
Le supplément originaire : Une lecture de la conception husserlienne de l'agrammaticalité
}

Par AMAURY DELVAUX

FRS-FNRS, Université de Namur

Ne se réappropriant pas la conceptualité de la phénoménologie transcendantale pour en utiliser la force heuristique à la résolution de problèmes d'ordre langagier et littéraire, Jacques Derrida a, cependant, mis au jour la plupart des concepts notoires de son œuvre - trace, différance, supplémentarité à partir d'une lecture extrêmement rigoureuse, située à la limite de l'interprétation et du commentaire ${ }^{1}$, de textes de la phénoménologie husserlienne. Si l'on regarde les écrits que Derrida consacre à ces textes, on constate que très souvent domine la thématique du langage. Plus précisément, Derrida s'attèle, d'une part, à une analyse poussée de la détermination phénoménologique du langage ordinaire et d'autre part, à un traitement attentif de la question du langage transcendantal - ce langage grâce auquel le phénoménologue manifesterait (Kundgeben) à autrui les résultats de ses descriptions phénoménologiques. Cette lecture qui trouve, sans doute, sa meilleure expression dans le court ouvrage La voix et le phénomène, se concentre avec insistance sur la première des Recherches logiques et ce malgré un intérêt pour un très grand nombre des textes husserliens ${ }^{2}$. Dans cet

${ }^{1}$ J. Derrida, La voix et le phénomène, Paris, PUF, coll. « Quadrige », 2009 ( $1^{\mathrm{e}}$ édition 1967), p. 20.

${ }^{2}$ L'analyse réalisée dans La voix et le phénomène est, dans une certaine mesure, redevable du travail que Derrida effectue au sujet des paragraphes 114 à 127 des Idées I. En effet, il serait possible de lire dans ce travail les premiers arguments en faveur du déploiement des concepts de différance et de supplémentarité en se focalisant sur la trame que tissent entre eux les vécus pré-expressifs et les vécus expressifs. $C f$. J. Derrida, « La forme et le vouloir-dire. Notes sur la phénoménologie du langage », in Marges de la philosophie, Paris, Éditions de Minuit, coll. « Critique », 1972, p. 185-207. 
ouvrage, les distinctions essentielles que cette première recherche expose font l'objet d'une étude dense et exhaustive. En effet, la mise à mal de la distinction capitale entre indice (Anzeichen) et expression (Ausdruck) n'intervient qu'à la fin de l'ouvrage ${ }^{1}$ et forme la conclusion d'une argumentation dont la complexité exige d'en saisir parfaitement chacune des étapes.

La quatrième recherche logique ne bénéficie pas d'une analyse comparable de la part de Jacques Derrida. Au contraire, le destin de la morphologie a priori des significations, développée dans cette recherche, se trouve, dès l'introduction de La voix et le phénomène, sèchement et définitivement réglé. Derrida dénonce avec sévérité la réduction de la grammaire pure à «l'a priori logique du langage $»^{2}$. Par là, cette grammaire pure ne concerne pas l'entièreté du langage mais exclusivement le langage de la connaissance scientifique. Et chaque fois que Derrida se réfère à cette grammaire pure logique, il ne manque jamais l'occasion de citer le passage où Husserl semble en reconnaître le caractère restreint :

Mais, dans la mesure où il ne peut nullement être affirmé que la morphologie pure des significations englobe tout l'a priori grammatical dans son universalité, puisque par exemple les relations de communications entre sujets psychiques, si importantes pour la grammaire, comportent un a priori propre, l'expression de grammaire pure logique mérite la préférence ${ }^{3}$.

Derrida reproche donc à Husserl d'avoir conçu sa grammaire pure exclusivement dans l'horizon d'une théorie de la connaissance - théorie qui, selon Derrida, relève encore de l'histoire de la métaphysique. De cette façon, Husserl retomberait dans les travers métaphysiques desquels il prétendait s'être dégagé en plaçant, dès le paragraphe 7 de l'introduction aux Recherches logiques, ses analyses sous l'impératif de la neutralité métaphysique. La position derridienne à l'égard de la grammaire pure husserlienne n'évoluera guère ; on en veut pour preuve cette phrase prononcée lors de la célèbre conférence de 1971, Signature événement contexte :

\footnotetext{
${ }^{1}$ Notons, toutefois, comme nous aurons l'occasion de le voir au cours de notre étude, que certains arguments importants, permettant déjà de faire sauter la distinction expression-indice, sont déployés dès les chapitres 2 et 3 . Toutefois, nous proposerons, ici, d'étudier comment les analyses derridiennes de la temporalité husserlienne viennent croiser les analyses dévolues au sens noématique, à l'idéalité.

2 J. Derrida, La voix et le phénomène, op.cit., p. 7.

${ }^{3}$ E. Husserl, Recherches logiques, t. II/2: Recherches pour la phénoménologie et la théorie de la connaissance, Paris, PUF, coll. « Épiméthée », 1961, p. 136.
} 
Husserl précise qu'il s'agit bien ici, à ses yeux, de grammaire pure logique, c'est-à-dire des conditions universelles de possibilité pour une morphologie des significations dans leur rapport de connaissance à l'objet possible, non pas d'une grammaire pure en général, considérée d'un point de vue psychologique ou linguistique ${ }^{1}$.

Néanmoins, en dépit de ses vues extrêmement critiques, Derrida reconnaît, dans un entretien avec Julia Kristeva publié en 1968, toute la centralité dans La voix et le phénomène de cette grammaire pure logique :

J'ai essayé d'indiquer ailleurs les conséquences qui lient toute la phénoménologie à ce privilège de l'expression, à l'exclusion de l'« indication » hors de la sphère du langage pur (de la «logicité » du langage), au privilège nécessairement accordé à la voix, etc., et cela dès les Recherches logiques, dès ce remarquable projet de "grammaire pure logique"

Propos doublement énigmatique puisque, d'une part, ils tranchent avec le point de vue soutenu un an plus tôt et d'autre part, Derrida soutient, dans le dernier chapitre de La voix et le phénomène, que la grammaire pure logique n'aurait pu voir le jour sans la distinction, élaborée dans la première recherche, entre signification et objet. Il y aurait donc lieu de donner la primauté à la première recherche logique et non à la quatrième. Pourtant, cette quatrième recherche semble, aux dires de Derrida lui-même, avoir suscité quelque chose de notable au sein de son travail autour de la phénoménologie husserlienne du langage. Il n'est pas dénué de sens de faire l'hypothèse que la grammaire pure logique forme, aux yeux de Jacques Derrida, l'endroit d'une certaine déception ${ }^{3}$ quant au projet phénoméno-

${ }^{1}$ J. Derrida, Marges de la philosophie, op. cit., p. 381.

2 J. Derrida, Positions, Paris, Éditions de Minuit, Coll. « Critique », 1972, p. 44.

${ }^{3}$ Il conviendrait, en vue de saisir tant bien que mal le(s) éventuel(s) motif(s) de cette déconvenue, de suivre patiemment certaines étapes de l'argumentation derridienne dans La voix et le phénomène et de les renvoyer au chapitre 1 de la première section de Logique formelle et logique transcendantale, chapitre où Husserl explicite l'articulation des différents niveaux de la logique formelle. Sans doute que Derrida voyait dans la morphologie a priori des significations la possibilité d'une libération du langage à l'égard de la légalité phénoménale. Or, il n'en sera rien et ce parce que Husserl a voulu contrecarrer le reproche émis par Marty à l'égard de sa grammaire. Marty reprochait à Husserl de subordonner la logique à la grammaire. Or, pour Husserl, il s'agit de ne plus rejeter la grammaire pure du côté de la psychologie mais de l'inclure à l'intérieur de la logique formelle et ainsi de ne pas réduire la logique à la simple logique de la validité. Tout ceci pourrait ainsi relativiser la critique de 
logique husserlien. Dans une certaine mesure, cette hypothèse permet de mieux rendre compte de ce paradoxal mélange d'admiration et de critique à l'égard de la morphologie a priori des significations.

Dans notre propos, il sera question de comprendre, tant que faire se peut, la place et le rôle qu'occupe la grammaire pure logique au sein de la réflexion derridienne sur la phénoménologie de Husserl. Plus particulièrement, on s'interrogera sur la façon dont Derrida utilise l'agrammaticalité (Sinnlosigkeit) - les expressions dépourvues de signification, par exemple Vert est ou, Abracadabra - pour élaborer son concept fondamental de supplément. Pour ce faire, on procédera en trois temps.

En vue de décrire la spécificité du mouvement argumentatif avec lequel Jacques Derrida aspire à faire sauter la distinction entre expression et indication, on aura soin de montrer, dans un premier temps, par quels ressorts démonstratifs, Derrida parvient à faire de la signification expressive le supplément indispensable au sens noématique. Ensuite, dans un deuxième temps, on étudiera la distinction implicite, au texte de Derrida et à sa lecture interprétative des Recherches logiques, entre absence de signification (Bedeutungslosigkeit) et absence de sens (Sinnlosigkeit). On se concentra, dans un troisième et dernier temps, sur la façon dont, à partir de la lecture de la première recherche logique, l'absence d'objet donné à l'intuition (Gegenstandslosigkeit) devient, pour Derrida, la structure fondamentale du vouloirdire, de la signification (Bedeutung). Le deuxième temps nous donnera l'occasion de pointer les déplacements que Derrida effectue implicitement à l'égard de l'agrammaticalité husserlienne et le troisième et temps de notre argumentation celle de voir en quoi ces déplacements participent activement à l'élaboration du supplément originaire et par là, à la démonstration de l'enchevêtrement (Verflechtung) entre l'indice et l'expression

Préalablement à notre étude, il aurait été, sans doute, judicieux de décrire de façon détaillée le cadre conceptuel au sein duquel l'élaboration du concept de supplément prend place. Aussi, la description de ce cadre aurait, à n'en pas douter, à couper court aux critiques réductrices - voire malveillantes - dont la lecture derridienne de Husserl fait, à notre sens, trop souvent l'objet. En ce sens, il convient de signaler au moins, d'une part, l'enjeu pour la phénoménologie husserlienne de l'ouvrage La voix et le

Derrida à l'égard de la grammaire pure logique puisque celle-ci n'a jamais eu pour ambition de s'identifier à la grammaire des linguistes et des psychologues. $C f$. Fr. Dastur, "Husserl et le projet d'une grammaire pure logique », in Cahiers de philosophie ancienne et du langage de l'Université : Grammaire, sujet et signification, vol. I (1994), p. 91-92. 
phénomène, c'est-à-dire la mise entre parenthèses de l'existence mondaine par la réduction transcendantale et, d'autre part, son enjeu pour la philosophie de Derrida. L'enjeu de cet ouvrage peut se résumer de la façon suivante : montrer comment, à partir de la conception husserlienne du signe, il s'avère légitime de défendre une productivité de la couche expressive des vécus à l'égard de leur couche pré-expressive ou, pour le dire en termes saussuriens, une action productive essentielle du signifiant à l'encontre du signifié - cette production fondamentale, Derrida la nomme supplément originaire, trace. Ce sont ces deux enjeux centraux du texte de Derrida que nous allons de façon brève et concise exposer préalablement au traitement de la question du rôle de l'agrammaticalité au sein du développement du concept de supplément. Cela nous donnera l'occasion, d'une part, de définir deux concepts husserliens importants, ceux d'indice (Anzeichen) et d'expression (Ausdruck), et d'autre part de voir selon quel objectif Derrida interroge la distinction que Husserl établit entre ces deux concepts

\section{De la distinction entre indice et expression au supplément originaire}

Exacerbant la dimension langagière de la réduction phénoménologique, Jacques Derrida tente de mettre en question la pureté du plan transcendantal à partir duquel Husserl entend déployer ses analyses les plus fructueuses. Derrida s'autorise à une telle inflation du motif langagier de la réduction transcendantale en raison de la distinction non substantielle - cette distinction ne s'opérant qu'en vertu de la réduction transcendantale - devant séparer la phénoménologie transcendantale de la psychologie phénoménologique :

Une différence radicale demeure, qui n'a rien de commun avec aucune autre différence ; différence qui ne distingue rien en fait, différence qui ne sépare aucun étant, aucune signification déterminée; différence pourtant qui, sans rien altérer, change tous les signes et en laquelle seulement se tient la possibilité d'une question transcendantale ${ }^{1}$.

Puisque la réduction transcendantale n'introduit aucune différence substantielle, elle ne peut ressortir, aux yeux de Derrida, que d'une opération langagière particulière : l'autodestruction du langage ordinaire ${ }^{2}$. À défaut de cette

${ }^{1}$ J. Derrida, La voix et le phénomène, op. cit., p. 11. Nous soulignons.

2 Ibid., p. 13. Dès la première recherche logique, Husserl concède qu'en vue de déterminer le terme exprimant correctement un concept, il convient parfois de vio- 
autodestruction, c'est la question transcendantale de la phénoménologie que l'on condamne à rester lettre morte. Contrairement à ce que Husserl défend, Derrida refuse de décrire les processus transcendantaux de constitution à l'aide du langage ordinaire et ce quand bien même on en utiliserait les concepts de façon exclusivement métaphorique en les entourant de guillemets.

Une fois cette inflation du motif langagier de la réduction transcendantale justifié, Derrida entend effectuer un rapprochement qui, du point de vue de la phénoménologie de Husserl, est loin d'apparaitre évident. Dans le chef du philosophe français, il est question de rapporter la différence nonsubstantielle distinguant la psychologie phénoménologique et la phénoménologie transcendantale à la différence entre les deux modalités de la signification que sont l'indice et l'expression :

Ne serait-on pas déjà en droit de dire que toute la problématique future de la réduction et toutes les différences conceptuelles dans lesquelles elle se prononce (fait/essence, transcendance/immanence, et toutes les oppositions qui font système avec elle) se déploient dans un écart entre deux types de signes [l'indice et l'expression $]^{1}$ ?

En ce sens, ce qui se trouverait, par là, suspendu à la question de la distinction entre signification indicative et signification expressive, c'est bien la réduction transcendantale. Par conséquent, de la validité de la distinction entre indice et expression dépend la possibilité de la réduction transcendantale. Si cette distinction se voyait invalidée, la réduction transcendantale s'apparenterait à une opération vaine et arbitraire parce qu'impossible

Jacques Derrida a beau pointer l'importance du langage dans l'effectuation de la réduction transcendantale en raison de l'absence d'une différence substantielle entre psychologie phénoménologique et phénoménologie transcendantale, on n'appréhende pas aisément pour autant pour quel(s) motif(s) la question transcendantale de la phénoménologique - la réduction transcendantale - tiendrait sa possibilité de la validité de la distinction,

lenter le langage lui-même : «Ce faisant, nous prenons toutefois le terme d'expression dans un sens restreint dont le domaine de validité exclut bien des choses que l'on qualifie d'expression dans le langage ordinaire. C'est de cette manière que l'on est forcé, par ailleurs, de faire violence à la langue, lorsqu'il s'agit de fixer terminologiquement des concepts pour lesquels on ne dispose que de termes équivoques $»$. $C f$. E. Husserl, Recherches logiques, t. II/1 : Recherches pour la phénoménologie et la théorie de la connaissance, Paris, PUF, coll. « Épiméthée », 1961, p. 35.

${ }^{1} \mathrm{~J}$. Derrida, La voix et le phénomène, op. cit., p. 35. 
opérée par Husserl, entre l'indice et l'expression. Il convient d'exposer rapidement, afin de saisir ces motifs, comment Husserl entend différencier l'indice et l'expression

D'après Husserl, la véritable expression ne peut être atteinte qu'à l'intérieur du "discours solitaire » et ce parce que l'expression, au sein du soliloque, cesse d'indiquer (Anzeigen) à autrui ce que je souhaite lui exprimer. Curieusement, l'expression ne se révèle telle qu'elle est essentiellement uniquement hors de toute communication intersubjective. Dans le discours communicatif, les expressions font figure d'indices des vécus psychiques que l'on manifeste (Kundgeben) à autrui ; l'expression opère ici une fonction de manifestation - les vécus psychiques formant le contenu manifesté. Néanmoins, l'expression exprime bien quelque chose ou plutôt, devrions-nous dire qu'elle désigne (Hinzeigen) le sens qu'un discours exprime. Alors que dans la communication, l'attention se porte sur les mots afin de manifester au mieux le vécu psychique que l'on cherche à communiquer, dans le discours solitaire, l'attention se détourne du mot pour se focaliser exclusivement sur la signification. Par conséquent, l'expression se trouve définie par Husserl en tant que « signe signifiant ».

En vertu de cette définition pour le moins singulière de l'expression, Jacques Derrida, soucieux de clarifier la démarcation husserlienne de l'expression par rapport à l'indice, proposera de traduire Bedeutung non plus par «signification » mais par «vouloir-dire ». En recourant à cette traduction, Derrida tente de prévenir le malentendu d'après lequel, suivant l'envers négatif de la définition husserlienne de l'expression, il existerait des signes non-signifiant. En ce sens, avec cette traduction particulière de la Bedeutung husserlienne, il s'agit dans le chef de Derrida, premièrement, d'éviter l'assimilation de la signification au renvoi indicatif, à l'indication et, deuxièmement, de ne pas faire de l'indice autre chose qu'une modalité spécifique du signe. Bien qu'il ne signifie rien — il ne veut rien dire —, l'indice demeure essentiellement un signe.

La signification, en sa fonctionnalité, n'opère pas de la même manière que l'indication: "Le signifier n'est pas une espèce de l'être signe au sens de l'indication (Anzeige) ${ }^{1}$. Contrairement à l'indication, la signification n'entend pas renvoyer, par l'intermédiaire d'une motivation associative, de l'existence d'une chose à la conviction ou présomption d'existence d'une autre. De cette façon, "là où une chose renvoie à une autre, là où une conviction de l'existence de l'une motive empiriquement (donc d'une

${ }^{1}$ E. Husserl, Recherches logiques, t. II/1 : Recherches pour la phénoménologie et la théorie de la connaissance, op. cit., p. 27. C'est Husserl qui souligne. 
manière contingente, non d'une manière nécessaire) celle de l'existence de l'autre ${ }^{1}$, nous avons affaire à de l'indication. De ce fait, dans l'action de désignation (Hinzeigen) propre à l'expression, «l'existence du signe ne motive pas l'existence, ou, plus précisément, notre conviction de la l'existence de la signification ». Et Husserl de continuer :

Ce qui doit nous servir d'indice (signe distinctif) doit être perçu par nous comme existant. Cela vaut bien pour les expressions dans le discours communicatif, mais non pour les expressions dans le discours solitaire ${ }^{2}$.

En dehors de sa fonction communicative, lorsque, dans le silence du monologue intérieur, elle se révèle enfin à elle-même, l'expression ne s'apparente aucunement à un quelconque existant. Plutôt que de vouloir mettre en lumière l'écart séparant les deux concepts liés au terme "signe ", Husserl entend prévenir d'une conception faisant de la signification un renvoi indicatif. La signification expressive - l'expression — renvoie au sens exprimé par un discours à la manière dont un panneau de signalisation indique la présence de travaux sur la route; elle n'indique (Anzeigen) pas ; elle désigne (Hinzeigen) !

Suivant attentivement la manière par laquelle Husserl est parvenu à séparer l'indice de l'expression, Derrida va trouver des recours argumentatifs l'autorisant à faire reposer la réduction transcendantale sur la distinction de ces deux modalités du signe. Ainsi, aux yeux de Derrida, la catégorie de l'existence mondaine - la factualité - apparaît, dans l'argumentation de Husserl, comme étant un facteur discriminant - si ce n'est pas le facteur discriminant - entre l'indice et l'expression: «Ce qui doit nous servir d'indice (signe distinctif) doit être perçu par nous comme existant $»^{3}$. Or, puisque la réduction transcendantale, comme on le sait, ne consiste pas en une mise à distance du monde mais plutôt en une neutralisation de sa thèse d'existence et de toute thèse d'existence possible, l'indice, en tant qu'il concerne uniquement des faits psychiques, s'identifie, de ce fait, à tout ce que la réduction transcendantale entend mettre entre parenthèses :

Ayant son origine dans les phénomènes d'association, liant toujours des existences empiriques dans le monde, la signification indicative [l'indice] couvrira, dans le langage, tout ce qui tombe sous le coup des « réductions» :

\footnotetext{
${ }^{1}$ Ibid., p. 32

${ }^{2}$ Ibid., p. 41.

${ }^{3}$ Ibid.
} 
la factualité, l'existence mondaine, la non-nécessité essentielle, la nonévidence ${ }^{1}$, etc.

Par l'intermédiaire d'une assimilation - que l'on pourrait peut-être jugée hâtive - de l'indice à la factualité, Derrida lie ainsi le destin de la réduction transcendantale au bien-fondé de la distinction entre les deux espèces du signe. Ne pas pouvoir isoler totalement l'expression en tant que telle de l'indice signerait, selon la lecture derridienne de la phénoménologie de Husserl, l'incomplétude de la réduction transcendantale et, par là, son échec.

Ainsi se présente ce que met en jeu, pour la phénoménologie, le court ouvrage de Jacques Derrida. Pris en lui-même, cet ouvrage semble mettre à mal le projet phénoménologique en ses principes fondateurs. En effet, afin d'invalider la distinction entre indice et expression, Derrida ira jusqu'à remettre en cause le principe des principes - le primat de la perception - à partir une lecture critique des Leçons pour la conscience intime du temps de Husserl. Plus précisément, ce que Derrida entend réfuter, par la mise en question du principe des principes de la phénoménologie, c'est l'idée husserlienne d'après laquelle les actes impliqués dans le monologue intérieur seraient vécus de façon immédiate et instantanée. Or, contrairement à cette idée soutenue par Husserl, ces actes ne peuvent être vécus qu'à la condition d'être indiqués. En ce sens, il s'avère erroné d'affirmer - comme le fait Husserl - l'absence de toute communication à l'intérieur du soliloque : lorsque je me parle à moi-même, je me communique bien quelque chose, je manifeste un contenu dont $\mathrm{j}$ 'ignorais la présence avant sa manifestation, sa communication.

S'il est vrai qu'avec la mise en cause de l'idée d'une présence à soi immédiate du vécu, Derrida entend plutôt réfuter l'absence d'une communication de soi à soi dans l'espace silencieux du monologue intérieur - ce n'est pas pour rien que, dès l'introduction de l'ouvrage de 1967, Derrida évoque les problèmes de l'intersubjectivité et de la temporalisation en tant qu'une non-présence semble prendre, dans le cadre de ces problèmes, une valeur constituante - , Derrida ne peut manquer d'interroger sérieusement l'impossibilité, défendue par Husserl, de rétroactions productives des vécus expressifs sur les vécus pré-expressifs tels les vécus perceptifs ou encore prédicatifs. Interrogation qu'il n'était parvenu qu'à effleurer dans son étude minutieuse $^{2}$ des passages que les Idées I consacrent aux vécus expressifs et à

${ }^{1}$ J. Derrida, La voix et le phénomène, op. cit., p. 34-35.

2 J. Derrida, «La forme et le vouloir-dire. Notes sur la phénoménologie du langage », in Marges de la philosophie, op. cit., p. 185-207. 
leurs relations aux autres vécus. On rejoint, par conséquent, l'enjeu pour la philosophie derridienne de l'essai La voix et le phénomène.

Dans une étude publiée dans La revue internationale de philosophie en 1967, Derrida n'avait pu que relever l'argumentation dogmatique par laquelle Husserl neutralisait toute productivité de la couche expressive :

Il faut bien, en fait, que l'ordre systématique du vouloir-dire impose de quelque manière son sens au sens, lui dicte sa forme, l'oblige à s'imprimer selon telle ou telle règle, syntaxique ou autre. Et ce « en fait» n'est pas une nécessité empirique parmi d'autres, on ne peut le mettre entre parenthèses pour poser des questions transcendantales de droit, puisque le statut du vouloir-dire ne peut être fixé sans qu'on détermine du même coup celui du sens. La mise entre parenthèses de ce «fait» est une décision quant au statut du sens en général dans ses rapports avec le discours. Elle ne dépend pas de la phénoménologie, elle l'ouvre d'un geste non-critique'.

Plutôt que de déclarer, dans un geste opposé et non-critique, l'imparfaite diaphanéité du signifiant (l'expression significative) à l'encontre du signifié (le sens), Derrida entend déceler une situation analytique chez Husserl par laquelle il va pouvoir, sans aller contre l'intention expresse du penseur allemand, mettre à mal l'improductivité de l'expression. Cette situation analytique, Derrida la trouve avec le soliloque à partir duquel Husserl dégage l'expression authentique. Cette mise à mal de la distinction entre indice et expression passe, comme on va le voir, par la démonstration de l'impossibilité d'une purification complète des significations indicatives au sein de l'espace purement expressif. Une des étapes de cette démonstration consiste à démontrer le nécessaire fonctionnement de la communication de soi à soi dans le silence du monologue intérieur.

\section{L'expression : supplément indispensable à la présence à soi du Sinn}

Dans l'introduction à L'origine de la géométrie, les concepts de trace et de supplément avaient émergé d'une étude minutieuse de la constitution des objectivités idéales. La voix et le phénomène propose de faire advenir ces mêmes concepts par l'entremise d'une réflexion faisant s'entrecroiser les thèmes de la signification et de la temporalité. Peut-être ne fait-on pas assez souvent remarquer la fonction stratégique de la lecture derridienne des Leçons pour la conscience intime du temps dans l'économie de l'interro-

${ }^{1}$ Ibid., p. 197. 
gation de la voix phénoménologique - ce que nous tenterons de faire ici en partie. Derrida semble mener cette interrogation selon deux itinéraires.

Le premier itinéraire - qui se déploie du chapitre 4 au chapitre $6-$ consiste à mettre en doute l'idée chère à Husserl selon laquelle, dans le soliloque, on ne se communique rien. Dès que l'expression entre dans une fonction de communication, elle se trouve nécessairement en commerce avec l'indication ; dans le soliloque, on se représente comme si l'on se communiquait quelque chose. Excellemment bien préparé par ce premier itinéraire, le second - le septième et dernier chapitre, Le supplément originaire s'emploie à démontrer que tout énoncé exprimé, bien qu'il trouve son sens remplissant (erfüllende Sinn), compose nécessairement avec une absence d'objet à l'intuition (Gegenstandslosigkeit). Comme annoncé ci-dessus, on se concentrera davantage sur ce second itinéraire puisque, dans celui-ci, la grammaire pure s'y trouve mobilisée de façon originale. De façon préliminaire, nous rappelons à nouveau qu'afin d'expliciter plus clairement la séparation husserlienne entre l'indice et l'expression, Derrida traduit, comme on l'a déjà indiqué, le terme allemand de Bedeutung non pas par « signification» mais par «vouloir-dire ». Assurément, Derrida l'a magnifiquement vu, la signification telle que la conceptualise Husserl ressort d'un acte volontaire : «la source de sens en général est toujours déterminée comme l'acte d'un vivre, comme l'acte d'être vivant, comme Lebendigkeit $»^{1}$. Et plus loin :

Car si l'intentionnalité n'a jamais voulu dire volonté, il me semble que dans l'ordre des vécus d'expression [...] conscience intentionnelle et conscience volontaire soient synonymes aux yeux de Husserl ${ }^{2}$.

Cette option traductive trouve, d'après Derrida, sa justification ultime dans la différenciation qui intervient treize ans plus tard entre le sens et la signification : « et elle [la distinction entre Sinn et Bedeutung] confirme [sa]

${ }^{1}$ J. Derrida, La voix et le phénomène, op. cit., p. 9.

${ }^{2}$ Ibid., p. 40. Derrida va même jusqu'à faire de la phénoménologie un volontarisme transcendantal et ce parce que tout vécu intentionnel peut venir se refléter dans un vécu expressif. Par là, le concept husserlien d'intentionnalité resterait prisonnier de la métaphysique. En ce sens, on comprend mieux pourquoi Derrida s'intéressera, quelques années plus tard, aux actes de langages de Austin et pourquoi, dans le cadre de cet intérêt, Derrida remobilise la phénoménologie husserlienne et plus particulièrement l'agrammaticalité telle que la développent la première et quatrième recherche logique. Cf. J. Derrida., « Signature événement contexte », in Marges de la philosophie, op. cit., p. 365-393. 
lecture ${ }^{1}$. Nous rappelons ici ces deux «présupposés » de lecture car nous aurons à cœur de montrer en quel sens ils permettent à Derrida d'atteindre plus facilement l'objectif — déjà mentionné ci-dessus — qu'il se fixe : démontrer l'irréductibilité de la signification indicative et ainsi fixer définitivement l'enchevêtrement (Verflechtung) entre indice et expression.

L'argumentation débute - au chapitre 3 - par un questionnement de la validité des deux arguments mis en avant par Husserl afin d'assurer la pureté expressive du soliloque : le caractère secondaire et contingent de la représentation dans le cas du monologue intérieur et l'inutilité d'une communication de soi à soi. Derrida vient brouiller la distinction que Husserl opère, à l'intérieur du langage, entre réalité et fiction : il existerait un langage fictif et un effectif. Derrida montre assez facilement qu'une telle différenciation dans le langage s'avère plus que bancale. En effet, étant donné que le signe en général - tant l'expression que l'indication - ne peut, par essence, s'apparenter à un événement empirique ayant lieu une seule et unique fois, il doit être identifiable par-delà les déformations empiriques de son inscription matérielle. Il doit maintenir son identité par le biais de sa possibilité d'être répété. En ce sens, Derrida note avec justesse :

En raison de la structure originairement répétitive du signe en général, il y a toutes les chances pour que le langage «effectif» soit aussi imaginaire que le discours imaginaire ; et pour que le discours imaginaire soit aussi effectif que le discours effectif. Qu'il s'agisse d'expression ou de communication indicative, la différence entre réalité et représentation, entre le vrai et l'imaginaire, entre la présence simple et la répétition a toujours déjà commencé à s'effa$\operatorname{cer}^{2}$.

Derrida réitère ce raisonnement lorsqu'il aborde l'idéalité du langage idéalité du signifiant et du signifié voire, dans certains cas, de l'objet :

Mais cette idéalité n'est que le nom de la permanence du même et la possibilité de sa répétition, [...]. Elle dépend toute entière de la possibilité d'actes de répétition ${ }^{3}$.

En ce sens, au sein du langage, on ne peut concevoir une séparation claire et nette entre la réalité et la représentation. D'après Derrida, la réalité du signe tient entièrement à son caractère représentationnel. Pour qu'un signe soit

${ }^{1}$ J. Derrida, La voix et le phénomène, op. cit., p. 21.

${ }^{2}$ Ibid., p. 60.

${ }^{3}$ Ibid., p. 61. 
réellement un signe en tant que tel, il doit pouvoir être répétable à l'infini. Ainsi, puisque l'ensemble des actes de signification se trouve pris dans une structure répétitive, tout discours s'accompagne nécessairement d'une représentation du sujet parlant et ce puisque, dans le discours solitaire, on se parle à soi-même. Voilà donc comment, dans un premier temps, Derrida s'y prend afin de remettre en cause l'absence d'indice au sein du soliloque. Dans un second temps, Derrida s'attèlera - dans le fameux chapitre 5 Le signe et le clin d'œil - à contrecarrer l'idée husserlienne d'une présence à soi immédiate du vécu. Invalider cette idée revient, comme on l'a déjà signalé, à prouver la nécessité d'une communication de soi à soi; le sujet doit se communiquer à lui-même la présence de son vécu. C'est du moins l'un des enjeux du chapitre 5 que l'on peut dégager à la lecture des dernières précisions du chapitre 4.

Dans le cadre du problème qui nous occupe, on s'enquerra de pointer de quelle manière les acquis du chapitre 5 se trouvent mobilisés afin de démanteler la parfaite transparence de l'expression à l'égard du sens qu'elle manifeste. Ce démantèlement constitue, à nos yeux, un des objectifs escomptés du chapitre 6, La voix qui garde le silence, bien qu'il s'agisse également de battre en brèche plus fortement encore la thèse husserlienne soutenant l'inutilité de l'indication dans le silence du monologue intérieur. Par là, l'enjeu principal de toute cette réflexion consiste à montrer qu'une écriture, une trace habite nécessairement et constamment cette voix silencieuse.

Dans ce chapitre 6, Derrida entend ici montrer en quoi le « s'entendreparler » de la voix phénoménologique - constitutive de l'idéalité de l'objet - ne forme pas une auto-affection pure. Pourquoi l'expression pure l'expression hors de sa fonction communicative ; la voix phénoménologique - forme-t-elle l'expédient par lequel l'idéalité trouve à se constituer ? Cela tient à l'essence de la parole : le s'entendre-parler. C'est un trait essentiel de la parole que «je m'entende dans le temps parler ${ }^{1}$. Par là, le phonème au sens phénoménologique est le plus idéal des signes et ce parce qu'il n'emprunte rien à la mondanéité. Du fait de sa structure autoréférentielle, ce « s'entendre-parler» - cette voix phénoménologique - se constitue en une auto-affection pure. Pure parce que, d'une part, elle se déplace dans le médium de l'universalité puisqu'elle mobilise des signifiés idéaux et ainsi répétables et, d'autre part, elle ne passe par aucun dehors, par aucune altérité. De là vient la capacité de la voix phénoménologique à se mouvoir

${ }^{1}$ Ibid., p. 92. 
dans l'universalité, à pouvoir assurer la possibilité d'une reproduction de l'idéalité en faisant l'économie de tout renvoi indicatif.

Parler à quelqu'un, c'est sans doute s'entendre parler, être entendu de soi, mais aussi et du même coup, si l'on est entendu de l'autre, faire que celui-ci répète immédiatement en soi le s'entendre-parler dans la forme même où je l'ai produit. Le répète immédiatement, c'est-à-dire reproduise l'auto-affection pure sans le secours d'aucune extériorité ${ }^{1}$.

Puisque l'efficace de la structure autoréférentielle de la voix phénoménologique s'étend jusqu'à la dimension intersubjective de son exercice, sens (signifié) et expression (signifiant) se trouvent dans une proximité telle que l'expression en vient à laisser transparaître, dans toute sa clarté, le sens. En raison de cette proximité absolue entre le sens et l'expression pure, Husserl peut conclure à la production improductive de l'expression quant au sens qu'elle exprime.

Par la suite, Derrida va placer cette analyse en face de sa lecture de la conception husserlienne de la temporalité. Pour rappel, Derrida avait montré en quoi la présence de l'impression originaire n'émergeait que de son rapport à la non-présence de la rétention - la rétention étant conçue, par Derrida, comme non-perception. De cette façon, le mouvement même de la temporalisation tient tout entier dans «cette trace rétentionnelle $»^{2}$. Derrida s'autorise, par un double motif, à exposer la voix phénoménologique aux conséquences de sa lecture des Leçons sur le temps de Husserl : d'une part, en raison de l'étoffe temporelle de la voix phénoménologique et d'autre part, parce que l'omni-temporalité propre aux objets idéaux et au sens constitue une modalité de la temporalité. Or, pour Derrida, l'impression originaire, telle que Husserl la conçoit, possède une structure auto-affective. Cette structure offre la possibilité à Derrida d'exposer la voix phénoménologique aux conséquences de sa lecture des Leçons pour une conscience intime du temps. Par conséquent, l'auto-affection de la voix phénoménologique - par laquelle le sens se temporalise - se voit elle-même toujours déjà fissurée par une trace, une non-présence. Elle souffre de façon originaire d'un espacement ; plus précisément, elle n'advient que dans l'espacement. Il ne saurait y avoir une pure intériorité de la parole préservée de tout rapport au dehors. Et Derrida de conclure ce développement décisif pour son argumentation :

${ }^{1}$ Ibid., p. 95.

${ }^{2}$ Ibid., p. 100. 
Celui-ci [le sens] étant, Husserl l'a reconnu, de nature temporelle, il n'est jamais simplement présent, il est toujours déjà engagé dans le mouvement de la trace, dans l'ordre de la signification. Il est toujours déjà sorti de soi dans la couche expressive du vécu ${ }^{1}$.

Après la présence de l'impression originaire à elle-même, c'est la présence à soi du sens qui se voit elle-même fissurée. Elle ne pourrait avoir lieu sans le supplément de l'expression qui l'extériorise, y compris quand le médium de l'expression relève de la voix phénoménologique.

En fin de course, Derrida parvient donc - d'une façon quelque peu indirecte - à affirmer le caractère essentiellement productif de l'expression quant au sens qu'elle exprime et ce quand bien même elle l'exprimerait par le plus idéal des signes, le signe phonique. L'expression suppléerait le sens qu'elle exprime ; c'est-à-dire que l'expression produirait ce à quoi il doit servir de représentant. Le sens ne saurait être totalement présent à lui-même sans le secours de l'expression. Or, et c'est là le point sur lequel Derrida a encore à s'opposer, l'expression reste pour autant transparente à elle-même puisqu'elle est habitée, de part en part, par le vouloir-dire - l'acte de signification - contrôlé par le sujet d'énonciation. Toutefois, le philosophe français envisageait d'aller plus loin. Que la temporalisation du sens doive passer par l'extériorité du monde ne suffit pas à prouver l'intrication indéfectible entre indication et expression - ce qui est, pour rappel, l'objectif poursuivi dans La voix et le phénomène. Certes, elle forme déjà un argument de taille. Toutefois, Derrida n'a démontré, pour l'instant, que la nécessaire suppléance de l'expression - et de son vouloir-dire - au sens noématique qu'il extériorise.

Il y a pour le moins quelque chose de particulièrement déroutant dans cette thèse d'une suppléance indispensable de la signification expressive à l'égard du sens noématique qu'elle manifeste. Déroutante, cette thèse l'est, au-delà de son caractère subversif, parce qu'elle s'élabore sur les bases d'un texte encore ignorant de la distinction, intervenant plus tardivement, entre Sinn et Bedeutung. Pourtant, à suivre Derrida lisant les Recherches, le sens (Sinn) est toujours déjà pris dans l'ordre de la signification (Bedeutung). Défendre une telle thèse à partir de l'exposé des Recherches logiques c'est clairement affirmer, au sein de cet exposé, l'existence d'une différence entre le sens et la signification. En s'appuyant sur ce dernier point, il sera question, dans le point suivant, de voir en quoi il pourrait exister au sein du texte husserlien, à suivre la lecture derridienne, une différence implicite entre

${ }^{1}$ Ibid., p. 101. 
Bedeutungslosigkeit et Sinnlosigkeit. Compte tenu de l'identification explicite du sens à la signification, Husserl ne devait pas faire le départ entre absence de signification et sens au sein des Recherches. Or, c'est justement ce que ne peut sous-entendre la lecture des Recherches par Derrida, qui envisage cet ouvrage au prisme de la distinction entre Sinn et Bedeutung.

\section{La différence entre Sinnlosigkeit et Bedeutungslosigkeit: une conséquence implicite de la traduction derridienne de Bedeutung par vouloir-dire}

Il est très instructif de voir comment Husserl s'emploie à élaborer la distinction - fondamentale pour sa grammaire pure - entre sens (Sinn) et nonsens (Unsinn). Au paragraphe 12 de la $4^{\mathrm{e}}$ Recherche logique, il rappelle la différence qu'il avait établie au paragraphe 15 de la $1^{\text {re }}$ Recherche entre sens et contresens. Cette différence tient essentiellement au fait que, dans le cas du contresens, l'on pouvait conclure la thèse suivante : «À la signification carré rond ne peut correspondre aucun objet existant $»{ }^{1}$. Or, pour parvenir à une telle conclusion, il faut que l'expression possède une signification unitaire minimale - elle doit au moins permettre de viser un objet inexistant, fictif voire impossible :

Si nous ne pouvions pas comprendre ce que veut dire «cercle carré» ou « montage d'or » comment pourrions-nous conclure à l'absence d'objet possible? C'est ce minimum de compréhension qui nous est refusé dans l'Unsinn, dans l'a-grammaticalité du non-sens ${ }^{2}$.

Plus précisément, dans des expressions agrammaticales, il ne s'agit plus d'une incompatibilité avec l'objet — comme c'est le cas avec des expressions absurdes - mais d'une incompatibilité avec la représentation. Avec les expressions dépourvues de signification unitaire, nous ne sommes en présence que de "représentation indirecte ». Voilà donc comment Husserl entend distinguer sens et non-sens.

L'on peut ressentir une certaine déception à l'issue de l'explication que nous livre ici Husserl. En effet, comme le déclare Husserl lui-même : "Cette opposition entre sens concordant objectivement, c'est-à-dire sur le plan de la signification, et contresens (Widersinn) est - grâce à notre

${ }^{1}$ E. Husserl, Recherches logiques, t. II/2 : Recherches pour la phénoménologie et la théorie de la connaissance, op. cit., p. 121.

${ }^{2}$ J. Derrida, La voix et le phénomène, op. cit., p. 107. 
définition des concepts - nettement séparée de l'opposition entre sens et non-sens $»^{1}$. Par conséquent, dans le chef de Husserl, il a été question, avec sa grammaire, semble-t-il, moins de départager le sens du non-sens que d'éviter de confondre le contresens avec le non-sens. De plus, le père de la phénoménologie ne semble pas éprouver la moindre difficulté à l'égard de la détermination du non-sens :

Le non-sens, chaque fois que l'on s'écarte de ces formes normales [définies par la morphologie a priori des significations], saute aux yeux d'une manière si immédiate que nous ne pouvons guère, dans la pratique de la pensée et de la parole, tomber dans de tels égarements ${ }^{2}$.

Par conséquent, Husserl identifie l'agrammaticalité au non-respect des catégories de la signification, par la transgression des frontières que ces catégories dessinent. Pourtant, Husserl avait dû reconnaître que, dans certains cas, cette transgression ne débouchait nullement sur des propositions dénuées de sens ou contradictoires. Et il importe de mettre en relief cette dernière stratégie argumentative de Husserl.

Énonçons très brièvement les traits saillants de cette morphologie $a$ priori des significations en vue d'aborder la manière dont Husserl répond aux objections que l'on peut formuler à l'encontre de cette morphologie. Pour qu'une proposition soit pourvue de signification, il faut que les différentes significations composant cette proposition s'organisent selon une morphologie a priori. Un jugement de type prédicatif n'aura une signification que s'il est construit selon la forme pure « ce $S$ est $P$ » $-S$ correspondant à la catégorie nominale et $P$ à la catégorie adjective. Par là, une matière adjective ne saurait s'immiscer à l'intérieur de la catégorie nominale sans, dans le même moment, rompre toute possibilité de signification unitaire pour la proposition.

Ces divers éléments en tête, il se révèle fort intéressant de voir comment Husserl traite des propositions du genre « si est une particule », c'est-àdire de ces expressions catégorématiques qui sont pourvues d'une signification et ce bien que les frontières des catégories de significations soient transgressées - dans notre exemple une matière conjonctive occupe la place normalement réservée à une matière nominale. Dans le chef de Husserl, les propositions de ce type, en plus d'être douées de sens, ne pâtissent d'aucune contradiction alors que l'expression - que nous prenions en exemple -

${ }^{1}$ E. Husserl, Recherches logiques, t. II/2, op. cit., p. 130.

${ }^{2}$ Ibid., p. 128. 
semble en elle-même contradictoire. La contradiction est ici évitée car la signification normale de l'expression syncatégorématique «si » a reçu une modification. Or, cette modification de signification relève pour Husserl simultanément de la normalité et de l'anormalité :

Du point de vue logique, tout changement de signification doit être considéré comme anomalie. L'intérêt logique qui porte sur les significations unitaires identiques exige la constance de la fonction de significations. Mais il est dans la nature des choses que certaines modifications fassent même partie de la structure grammaticale de toute langue ${ }^{1}$.

Et Husserl de conclure : «Ce qui est anormal au point de vue logique se présente alors comme sanctionné par la grammaire $»^{2}$.

Afin de circonscrire la portée de cette anormalité logique autorisée par la grammaire, Husserl recourt à diverses tactiques - par méthodologie, nous n'en retenons qu'une seule. Dans le cas des significations modifiées, il va défendre, en se référant à la suppositio materialis, que l'expression «si » ne délivre plus sa signification courante mais la représentation de cette signification. Et pour indiquer qu'il s'agit non de la signification normale mais de sa représentation, Husserl mentionne l'utilisation des fameux guillemets ou «d'autres moyens d'expression extra-grammaticaux » ${ }^{3}$. Tout ceci ne va sans rappeler la stratégie argumentative mobilisée par Husserl en vue de défendre l'absence d'une communication de soi à soi au sein du soliloque : je ne me communique rien; je me représente comme si je me communiquais quelque chose. Ici, aussi, semblerait pouvoir fonctionner l'argument derridien d'après lequel on ne saurait, à l'intérieur du langage, faire le départ entre la réalité et la représentation.

Derrida ne fait que relever, au passage, l'usage par Husserl de ces moyens extra-grammaticaux - les guillemets - lors de l'analyse de la constitution du flux temporel de la conscience. Pour des raisons stratégiques qui vont apparaître dans un instant, il préfère se focaliser sur la différence entre la Bedeutungslosigkeit ${ }^{4}$ et la Gegenstandslosigkeit ; différence décisive,

${ }^{1} I b i d .$, p. 116. Nous soulignons.

${ }^{2}$ Ibid.

${ }^{3}$ Ibid., p. 117.

${ }^{4}$ Dans le cadre des Recherches logiques, il faut prendre pour synonymes les termes allemands de Bedeutungslosigkeit et de Sinnlosigkeit. À notre connaissance, Husserl n'utilise qu'une seule fois le terme de Bedeutungslosigkeit - dans le paragraphe 15 de la première recherche — pour signifier, à notre sens, l'agrammaticalité. Toutefois, 
telle qu'elle s'établit initialement dans le paragraphe 15 de la première Recherche logique. Il importe de relever que, contrairement au terme choisi dans la $1^{\text {re }}$ Recherche logique, Husserl, dans la $4^{\mathrm{e}}$ Recherche, n'utilise pas, afin de signifier l'agrammaticalité, le terme allemand de Bedeuntunglosigkeit mais celui de Sinnlosigkeit. Or, à suivre la traduction derridienne de Bedeutung, Bedeutungslosigkeit signifie moins absence de signification qu'absence de vouloir-dire, et par conséquent, désigne l'indice. En ce sens, les énoncés dit bedeuntunglos ne seraient que des indices et, par là, resterait des signes dont la modalité de signification diffère de celle propre à l'expression : la désignation (Das Hinzeigen). Aussi, il convient de pointer que, conformément aux présupposés de la lecture derridienne des Recherches, Bedeutungslosigkeit ne s'avèrerait pas être, comme c'est le cas chez Husserl, le synonyme de Sinnlosigkeit. Bien qu'il lit les Recherches logiques, Derrida envisage déjà la notion de Sinn comme couvrant toute la sphère noématique et la Bedeutung toute la sphère de l'expression, à l'instar de ce qui est défendu au sein des Idées directrices.

Dès lors que l'extension du sens déborde absolument le vouloir-dire, le discours aura toujours à puiser son sens. Il ne pourra d'une certaine manière que répéter ou reproduire un contenu de sens qui ne l'attend pas pour être ce qu'il est. Le discours ne fera, s'il est ainsi, que porter au dehors un sens constitué sans lui et avant lui ${ }^{1}$.

Ainsi, aux yeux de Derrida, Sinnlosigkeit désignerait, pour le dire dans la terminologie des Recherches logiques, une absence de sens d'appréhension, autrement dit, un contenu de sensation dépourvu de toute appréhension intentionnelle, des data hylétiques inanimés intentionnellement tandis que Bedeutungslosigkeit définirait l'expression dénudée de vouloir-dire ; c'est-àdire l'indice. Or, comme on le sait, le sens d'appréhension, la matière (Materie), confère, à l'acte sa relation à l'objectité. Par conséquent, que Husserl parle, au sein de la $4^{\mathrm{e}}$ Recherche logique, de Sinnlosigkeit plutôt que de Bedeutungslosigkeit confirme son intention d'expliciter une morphologie a priori des significations capable de toujours conférer une relation à un objet - que celui-ci soit efficient, possible, impossible ou fictif. Certes, à strictement parler, Sinnlosigkeit signifie bien, dans le cadre du texte husserlien, une absence de sens linguistique. Néanmoins, dans l'espace de la

la lecture qu'effectue Derrida des Recherches logiques a, comme on va le voir, pour conséquence implicite d'invalider cette synonymie.

${ }^{1}$ J. Derrida, «La forme et le vouloir-dire. Notes sur la phénoménologie du langage », op. cit., p. 194. 
lecture derridienne des Recherches logiques, ce terme en vient à nommer également une absence de relation à l'objectité et ce en vertu du fait que, pour le philosophe français, Sinnlosigkeit ne dénote plus l'absence d'un sens linguistique - d'une signification - mais bien celle d'un sens noématique. De la même manière, Bedeutungslosigkeit ne signifie plus, chez Derrida, l'absence d'un sens linguistique — d'une signification - mais bien l'indication, l'absence d'un vouloir-dire ; c'est-à-dire un défaut à l'endroit de la volonté d'un sujet parlant à exprimer une certaine signification à l'aide d'une ou plusieurs propositions composées de mots. Ainsi, les énoncés dits bedeutungslos s'apparentent, du point de vue de Derrida, à des indices et, par là, à des signes.

Toutefois, Derrida ne pense pas effectuer ici une interprétation abusive de la Sinnlosigkeit telle que définie par la phénoménologie de Recherches logiques. En effet, on lit bien dans les Recherches logiques:

C'est dans la signification que se constitue le rapport à l'objet. Par conséquent, employer une expression avec sens, et se rapporter par une expression à l'objet (se représenter l'objet), c'est là une seule et même chose ${ }^{1}$.

Par là, une expression agrammaticale revient à n'avoir aucun rapport à un objet et ce en dépit du fait qu'avant les Idées directrices, Husserl ne sépare pas le sens (Sinn) - ce qui va conférer la relation à une objectité - de la signification linguistique. En ce sens, puisque, dans les Recherches logiques, Sinn et Bedeutung recouvrent, en raison de leur synonymie, une même réalité, Derrida pense, par là, démontrer que toute la théorie de la signification ainsi que toute la grammaire pure ont été élaborées en fonction de la possibilité de la relation à l'objet.

$\mathrm{Si}$, dans le cadre des Recherches logiques, les termes « signification» (Bedeutung) et « sens 》 (Sinn) — et par là les termes «Bedeutungslosigkeit » et «Sinnlosigkeit» - sont interchangeables, une lecture, prenant le parti de lire ces Recherches en tenant compte de la distinction entre ces deux termes, en arrive à faire les déplacements conceptuels importants que nous venons de pointer. À strictement parler, Bedeutungslosigkeit se traduit par l'absence de signification. Toutefois, Derrida s'écarte, comme on l'a vu, de cette traduction et préfère traduire ce terme allemand par l'absence de vouloir-dire, autrement dit, par l'indication et ce parce que l'indice se trouve caractérisé, par Derrida, en tant que signe dépourvu de vouloir-dire. De ce fait, une expression dite bedeutungslos ne se réduit pas à une simple flatus vocis. Elle

${ }^{1}$ E. Husserl, Recherches logiques, t. II/1, op. cit., p. 61. 
continue à appartenir à l'ordre des signes. Bedeutungslosigkeit cesse de renseigner l'absence de sens tant linguistique que noématique pour ne renvoyer qu'au défaut de vouloir-dire d'un signe, donc à l'indice. Elle marque le fait qu'un signe n'est pas animé d'une intention de signification; elle pointe un défaut du côté de la volonté — de l'intention ${ }^{1}$ — plutôt que du côté de la signification.

Comment convient-il, dans ce contexte interprétatif, de définir la Sinnlosigkeit ? Contrairement à la définition husserlienne, la Sinnlosigkeit ne constitue pas, selon Derrida, le synonyme parfait de la Bedeutungslosigkeit. $\mathrm{Du}$ fait que, chez Derrida, la Bedeutungslosigkeit fait référence à l'indication, à l'absence de vouloir-dire d'une expression, la Sinnlosigkeit va, à elle seule, désigner l'absence totale de sens tant linguistique que noématique. Dans le cas des expressions dites sinnlos, ce qui est pris en défaut c'est moins l'intention que la signification. Plus précisément, avec ce type d'expression, il existe bien une volonté de dire quelque chose avec l'expression mais le contenu que l'on souhaite exprimer s'avère, en lui-même, incompréhensible. Par là, il s'agit moins d'une défaillance de la signification que celle du sens noématique que la signification cherchait à exprimer. Cette situation tient au fait que, dans le chef du Husserl des Idées, la signification ne forme que le reflet linguistique d'un sens pré-linguistique - noématique - conférant une relation à une objectité. En conclusion, la Bedeutungslosigkeit renvoie à la déficience de la volonté tandis que la Sinnlosigkeit à celle du sens noématique.

En quoi cette différence, proprement issue de la lecture derridienne de Husserl, entre Bedeutungslosigkeit et Sinnlosigkeit peut-elle nous renseigner sur la stratégie de lecture déployée par Derrida? D'une certaine manière, cette différence amène à poser une question franchement complexe au Husserl des Recherches logiques, à savoir si la signification forme l'expédient principal de la relation à l'objet, cette signification se distingue-t-elle

${ }^{1}$ Selon Derrida, dans le cadre de l'étude des vécus expressifs, l'intentionnalité s'apparente à la volonté, à l'intentio augustienne: «Si l'intentionnalité n'a jamais voulu dire simplement volonté, il me semble bien que dans l'ordre des vécus d'expression (à supposer qu'il ait des limites) conscience intentionnelle et conscience volontaire soient synonymes au yeux de Husserl. Et si l'on en venait à penser comme Husserl nous y autorisera dans Ideen I - que tout vécu intentionnel peut en principe être repris dans un vécu d'expression, on devrait peut-être conclure que malgré tous les thèmes de l'intentionnalité réceptrice ou intuitive et de la genèse passive, le concept d'intentionnalité reste pris dans la tradition d'une métaphysique volontariste, c'est-à-dire peut-être simplement dans $l a$ métaphysique ». $C f$. J. Derrida, La voix et le phénomène, op. cit., p. 40. 
du sens d'appréhension ou de la matière ${ }^{1}$ (Materie) ? Ainsi, la question que Derrida adresse à la phénoménologie des Recherches peut se formuler en ses termes : existe-t-il réellement une indépendance absolue entre le vécu préexpressif et le vécu expressif? Ne partagent-t-ils pas une interdépendance dont aucun de ces types de vécus ne pourrait faire l'économie? Derrida formule, ainsi, à l'endroit des Recherches logiques la question qu'il adressait au Husserl des Idées directrices.

À bien y regarder, lire les Recherches logiques à l'aune d'une distinction entre le sens et la signification ne concernant lesdites Recherches permet à Derrida de démontrer plus aisément l'inévitable enchevêtrement (Verflechtung) entre les vécus pré-expressifs et les vécus expressifs, entre sens noématique et signification. Toutefois, en dépit de l'objectif de cette stratégie de lecture, celle-ci a au moins le mérite d'attirer l'attention du lecteur des Recherches logiques sur un aspect moins saillant de la définition husserlienne de l'agrammaticalité (Bedeutungslosigkeit ou Sinnlosigkeit). Par là, on tentera, afin de conclure ce troisième point, de montrer en quoi la distinction entre Bedeutungslosigkeit et Sinnlosigkeit, implicite à la lecture derridienne des Recherches, peut trouver une relative validation au sein même du texte de Husserl.

Partons d'une affirmation tenue par Husserl au paragraphe 15 de la Première recherche :

Une expression sans signification n'est par conséquent, à proprement parler, en aucune façon une expression; en mettant les choses au mieux, elle est une chose quelconque qui a la prétention ou l'apparence d'être une expression alors qu'à y regarder de plus près, il n'en est nullement ainsi ${ }^{2}$.

Une expression vierge de toute signification forme une chose ayant l'apparence d'une expression. Et cette apparence tient, comme le précise Husserl, à la façon dont une expression sans signification ou un ensemble formé de véritables expressions se présentent extérieurement. Par leur agencement

\footnotetext{
${ }^{1}$ On serait tenté d'avancer qu'au motif de la synonymie entre sens et signification, entre le sens d'appréhension et la signification expressive, il n'existe aucune sorte de différenciation mais, par là, on nierait peut-être le fait que perception et signification forment deux modalités à part entière de l'intentionnalité objectivante. Ou, devraiton dire que ces différentes modalités de l'intentionnalité - signification, imagination, perception - puisent à une même et unique source, le sens noématique, la matière intentionnelle (Materie) ?

${ }^{2}$ E. Husserl, Recherches logiques, t. II/1, op. cit., p. 61.
} 
extérieur, une expression ou un ensemble d'expressions peuvent apparaitre comme doués d'une signification.

Ici peut se dégager, au sein du texte même des Recherches logiques, une ligne de démarcation entre le sens (Sinn) et la signification (Bedeutung) et ce quand bien même une telle démarcation n'aurait pas cours dans ce texte. De l'expression sans signification, Husserl nous dit qu'elle forme une chose dont l'apparence nous ferait croire que l'on a affaire à une véritable expression. Et ce n'est qu'au prix d'un regard plus attentif — « à y regarder de plus près »-que sous l'apparente expression se dévoile une expression en défaut de signification, une expression inauthentique. Or, à propos du non-sens (Unsinn), Husserl précise, dans un passage déjà cité plus haut, que celui-ci saute immédiatement aux yeux. Contrairement à l'expression dépourvue de signification (bedeutungslos), l'expression a-sensée (sinnlos) nous apparaît directement en tant que telle. Le non-sens (Unsinn) s'avère plus simple à déceler que l'expression dépourvue de signification (bedeutungslos). Voilà ce qui pourrait fournir, à lire à la lettre les Recherches logiques, l'élément permettant de discriminer la Sinnlosigkeit de la Bedeutungslosigkeit.

Néanmoins, on ne saurait s'empêcher de formuler l'interrogation suivante : à quoi d'autre peut correspondre cette chose ayant l'apparence d'une véritable expression si ce n'est l'indice ? En effet, de la même façon qu'il a fallu être attentif afin de distinguer entre le signifier propre à l'indice et le signifier de l'expression, il convient d'adopter un regard attentif afin de percer l'inauthenticité d'une expression. On se retrouve dans une situation à peu près similaire à celle antérieure à la distinction effectuée entre l'expression et indice: en apparence, et avant analyse, ces deux termes semblent synonymes et, par là, recouvrir un même espace conceptuel, celui du signe signifiant.

Fort de la distinction, sous-jacente à la lecture derridienne des $R e$ cherches, entre Bedeutungslosigkeit et Sinnlosigkeit, il faut à présent voir en quoi Derrida invalide la distinction que Husserl élabore entre une absence d'objet susceptible de remplir intuitivement et de façon satisfaisante un énoncé et l'agrammaticalité (Bedeutungslosigkeit ou Sinnlosigkeit). Encore une fois, Derrida va s'autoriser un déplacement conceptuel important en regard du texte même des Recherches puisqu'il va, ni plus ni moins, étendre la Gegenstandslosigkeit réservée normalement et exclusivement aux énoncés absurdes ou contradictoires à l'ensemble des propositions significatives. Et c'est par l'intermédiaire d'une telle extension que Derrida parviendra à faire sauter la distinction essentielle entre expression et indice. 


\section{La Gegenstandslosigkeit : structure fondamentale de la signification expressive}

La lecture du dernier chapitre de La voix et le phénomène s'avère des plus déconcertantes et ce parce qu'il regorge de thèses capitales pour la philosophie de la déconstruction. Déconcertante, cette lecture l'est également car ces thèses vont à rebours de toute l'histoire de la métaphysique ; elles veulent inhumer le rapport à ma propre mort dont cette histoire n'a cessé de vouloir contenir la résonnance :

La possibilité du signe est ce rapport à la mort. La détermination et l'effacement du signe dans la métaphysique sont la dissimulation de ce rapport à la mort qui produisait pourtant la signification ${ }^{1}$.

Fidèle à son précepte selon lequel «il n'y a aucun sens à se passer des concepts de la métaphysique pour ébranler la métaphysique $»^{2}$, Derrida va donc prendre appui sur le concept précieux - nous verrons pourquoi - de la grammaire pure husserlienne: l'absence d'objet donné à l'intuition (Gegenstandslosigkeit). Cette grammaire forme, à tout point de vue, un avatar de l'histoire de la métaphysique puisqu'elle s'apparente à une morphologie a priori de la signification. Or, on sait que la forme - morphè, eidos, form - constitue l'un des concepts phares de la métaphysique. Derrida a ouvertement rapproché ce concept du thème de la présence. En conséquence, il nous faut exposer certains points de cette morphologie afin d'y déceler par quel biais Derrida parvient à rétablir le signe à l'intérieur de la métaphysique.

Le problème de l'absence d'objet donné à l'intuition est travaillé par Husserl par l'intermédiaire des propositions dites absurdes ou contradictoires (widersinnig). Ces dernières possèdent bien une signification unitaire, un sens remplissant (erfüllende Sinn). Cependant, elles sont dites dépourvues d'objet à l'intuition (gegenstandslos); ce qui ne veut pas dire qu'elles sont sevrées de tout rapport à l'objet puisque la signification, pour rappel, forme une modalité de l'intentionnalité objectivante : «La purification du formel se règle sur un concept de sens lui-même déterminé à partir d'un rapport à l'objet $»^{3}$. Simplement, l'énoncé absurde ne trouvera jamais une intuition le

${ }^{1}$ J. Derrida, La voix et le phénomène, op. cit., p. 64.

2 J. Derrida, «La structure, le signe et le jeu dans le discours des sciences humaines », in L'écriture et la différence, Paris, Editions du Seuil, coll. « Points », 1967, p. 412.

${ }^{3}$ J. Derrida, La voix et le phénomène, op. cit., p. 115. 
remplissant de façon adéquate et, pour cela, il est qualifié de gegenstandslos. Il y a lieu ici de préciser les choses afin de couper court à tout malentendu. Quand Husserl affirme qu'avec les énoncés contradictoires, on se confronte avec des énoncés pourvus simplement d'un sens remplissant et non pas également d'une intuition capable de remplir adéquatement cet énoncé, cela ne contredit pas la thèse énoncée plus haut selon laquelle c'est dans la signification que se forme le rapport à l'objet. En effet, la proposition absurde, parce qu'elle s'avère compréhensible, pourvue d'une signification, déploie un rapport à l'objectité (Gegenständlichkeit) et ce parce qu'elle signifie un objet impossible, carré rond. Plus précisément, l'expression absurde ne revêt aucune relation à un objet pouvant faire face à la conscience - un Gegenstand; par contre, elle possède une relation à ce qui a le statut d'objet en général, l'objectité (Gegenständlichkeit). Ainsi, dans le chef de Husserl, l'absence d'objet à l'intuition (Gegenstandslosigkeit) n'équivaut pas à un défaut du côté de l'objectité (Gegenständlichkeit). Seulement la proposition incompréhensible (sinnlos ou bedeutungslos) peut être dite dépourvue de toute relation à l'objectité et donc, par là, à un objet capable de la remplir intuitivement de façon adéquate. Par conséquent, toute Widersinnigkeit s'accompagne nécessairement d'une Gegenstandslosigkeit mais pas d'une absence d'objectité

Partant de la subtile distinction husserlienne entre la Gegenstandslosigkeit et l'agrammaticalité (Sinnlosigkeit ou Bedeutungslosigkeit), Derrida va fonder son argumentation sur la thèse husserlienne d'après laquelle il fait partie de l'essence de l'expression que celle-ci puisse se passer de la présence à l'intuition de l'objet (Gegenstand) qu'elle vise. Que l'objet puisse faire défaut à la perception ne réduit pas l'expression à un simple flatus vocis. Celle-ci peut être comprise sans que pour autant il soit nécessaire que j'aie effectivement la perception hic et nunc des objets visés par l'énoncé. Cet énoncé s'avère intelligible y compris pour celui qui se trouverait à « une distance infinie dans le temps et dans l'espace ${ }^{1}$. Et Derrida de poursuivre :

L'absence totale du sujet et de l'objet d'un énoncé — la mort de l'auteur ou/et la disparition des objets qu'il a pu décrire - n'empêche pas un texte de vouloir-dire. Cette possibilité au contraire fait naître le vouloir-dire comme tel, le donne à entendre et à lire ${ }^{2}$.

${ }^{1}$ Ibid., p. 108.

${ }^{2}$ Ibid., p. 109. 
Par conséquent, Derrida étend la Gegenstandslosigkeit à l'ensemble des expressions significatives; elle n'est plus exclusivement réservée aux expressions absurdes ou contradictoires. Comme, pour Derrida, d'une part, la compréhension - l'intelligibilité - forme le critère par lequel la Widersinnigkeit se différencie de la Sinnlosigkeit et, d'autre part, cette intelligibilité de l'expression tient essentiellement à la possibilité de comprendre l'énoncé en dehors de son contexte d'énonciation, le philosophe français s'autorise une telle extension de la structure de la Gegenstandslosigkeit. Elle concerne l'expression en général et non plus, comme c'est le cas chez Husserl, uniquement les expressions absurdes ou contradictoires. Voilà donc le premier élément susceptible de venir contredire la distinction établie par Husserl entre indice et expression.

Le second élément - décisif — intervient, quant lui, à l'occasion de l'étude de l'expression «je». Cette expression fait partie du groupe des «expressions occasionnelles ». Ce groupe se caractérise par le fait inédit suivant: leur signification s'oriente vers tel ou tel objet en fonction de la situation de celui qui parle. On peut reconnaitre une expression occasionnelle à l'impossibilité d'y substituer une expression objective sans aboutir à quelques absurdités. De surcroît, Husserl admet que :

Dans le discours solitaire, la signification du je se réalise dans la représentation immédiate de notre personnalité, et c'est là que réside donc aussi la signification de ce mot dans le discours communicatif. [...]. Mais, comme toute personne, quand elle parle d'elle-même, dit je, ce mot possède le caractère d'un indice universellement efficient pour désigner cette situation ${ }^{1}$.

Derrida n'en demandait pas plus. En effet, la situation des expressions occasionnelles ne va pas sans poser problème, une fois l'absence d'objet donné à l'intuition (Gegenstandslosigkeit) instaurée en structure fondamentale du vouloir-dire. Que l'expression «je» ne puisse signifier en dehors du contexte de son énonciation signerait son incapacité à vouloir-dire, à signifier. Tout discours contenant l'expression «je» doit pouvoir être compris, en tant que discours, indépendamment de la présence du sujet de l'énonciation :

${ }^{1}$ E. Husserl, Recherches logiques, t. II/1, op. cit., p. 95. Nous soulignons. 
Lorsque je me dis à moi-même «je suis », cette expression, comme toute expression selon Husserl, n'a le statut de discours que si elle est intelligible en l'absence de l'objet, de la présence intuitive, donc ici de moi-même ${ }^{1}$.

Encore une fois, Derrida étend une situation particulière dont Husserl tentait de restreindre la portée, à la totalité des expressions faisant intervenir hic et nunc le sujet. Ce que Husserl réservait à l'exclusivité de la lecture de l'expression «je», Derrida en fait la structure «normale» de toute expression langagière :

Quand nous lisons ce mot « je» sans savoir qui l'a écrit, nous avons un mot, sinon dépourvu de signification, du moins étranger à sa signification normale ${ }^{2}$.

Étant donné que l'expression «je » ne peut être intelligible qu'à la condition expresse que l'objet je puisse à jamais disparaître, ce que Husserl jugeait relever de l'anormalité constitue la signification normale de l'expression «je»:

Ma mort est structurellement nécessaire au prononcé du Je. [...] L'énoncé « je suis vivant» s'accompagne de mon être-mort et sa possibilité requiert la possibilité que je sois mort; et inversement ${ }^{3}$.

Ainsi s'expose le second élément permettant de faire sauter la distinction entre indice et signification :

L'indication pénètre ainsi partout où dans le discours une référence à la situation du sujet ne se laisse pas réduire, partout où celle-ci se laisse signaler par un pronom personnel, un pronom démonstratif, un adverbe "subjectif» du type ici, là-bas, en haut, en bas, maintenant, hier, demain, avant, après ${ }^{4}$, etc.

Pourquoi cette référence à la situation du sujet ferait-elle entrer de façon inévitable de l'indication à l'intérieur de l'expression ? Que le je puisse ne jamais pouvoir faire l'objet d'une intuition ne signe pas définitivement le recours indépassable à l'indice. Or, pour Derrida, il ne peut en aller autrement. Puisque l'expression occasionnelle s'oriente en fonction de la

${ }^{1} \mathrm{~J}$. Derrida, La voix et le phénomène, op. cit., p. 111.

${ }^{2}$ E. Husserl, Recherches logiques, t. II/1, op.cit., p. 95.

${ }^{3}$ J. Derrida, La voix et le phénomène, op. cit., p. 113.

${ }^{4}$ Ibid., p. 110 
situation de l'énonciateur, elle mettrait en péril la différence entre Gegenstandslosigkeit et Bedeutungslosigkeit - traduite ici par « absence de vouloir-dire ». L'expression occasionnelle «je », séparée de la situation de celui qui l'énonce, relèverait davantage d'une certaine interprétation (Deutung), à l'instar en quelque sorte des significations des gestes corporels accompagnant notre parole. En ce sens, pour toute proposition devant impliquer la situation hic et nunc de l'énonciateur, le passage par l'indication s'avère inévitable afin de pouvoir être intelligible par tous. Puisque l'expression occasionnelle ne peut signifier sans une référence à la situation de son énonciation, il faut que cette situation soit indiquée lorsque celle-ci a cessé d'être présente. Toutefois, jamais il ne sera possible à celui qui entend ou lit une proposition avec une expression occasionnelle en dehors de ce contexte d'énonciation, de saturer ce contexte - si une telle saturation a jamais existé - qui n'est alors que seulement indiqué. Or, faisant de la Gegenstandslosigkeit la condition indispensable du vouloir-dire, Derrida conclut que, peu importe si le contexte d'énonciation est présent ou pas, l'indication a toujours contaminé l'expression une fois qu'elle inclut l'hic et nunc, l'Augenblick du sujet de l'énonciation ; celui-ci a toujours déjà commencé à s'absenter, à se manquer.

Toutefois, une philosophie faisant de la Gegenstandslosigkeit le facteur essentiel à tout vouloir-dire ne peut que nous pousser à formuler une telle interrogation : Que voulait de façon expresse signifier Husserl lorsqu'il préfère utiliser le terme de Bedeutungslosigkeit à la place de celui de Sinnlosigkeit ? Faut-il affirmer la synonymie de ces deux termes? L'option traductive optée par Derrida à l'encontre de la Bedeutung interdit d'envisager une telle synonymie. De surcroît, cette interdiction se trouve renforcée compte tenu du fait que les Recherches logiques sont lues par Derrida au prisme de la distinction plus tardive entre Sinn et Bedeutung. Mais, dans le cadre d'une analyse qui n'a pas encore fait le départ entre Sinn et Bedeutung - celle des Recherches logiques -, ne faut-il pas entendre par Bedeutungslosigkeit l'agrammaticalité au sens de la Sinnlosigkeit ? Car, lorsque, dans la $4^{\mathrm{e}}$ Recherche logique, il réaffirme la différence entre absence d'objet et absence de signification, Husserl ne parle plus de Bedeutungslosigkeit comme il l'avait fait dans la $1^{\text {re }}$ Recherche - mais de Sinnlosigkeit. Il ne faut pas s'y tromper. Quand Husserl distingue la contradiction du non-sens, il n'entend pas affirmer qu'avec le non-sens, nous avons affaire à des indices, à des parties sensibles de l'expression mais à des choses dont l'agencement extérieur nous fait penser qu'il s'agit de véritables expressions. Dans le chef de Husserl, Bedeutungslosigkeit ne désigne nullement l'indication mais bien l'absence de signification d'une expression ou d'un ensemble composé de 
véritables expressions. Resterait à s'interroger sur le statut que Husserl attribue à ces choses dont l'organisation extérieure nous incline à croire que l'on a affaire à une ou des expression(s) authentique(s).

En dépit de ces considérations, Derrida ne semble pas suivre cette lecture de la Bedeutungslosigkeit. Lorsqu'on lit le texte de Husserl par dessus l'épaule de Derrida - pour reprendre cette expression que Derrida utilise dans sa lecture de Foucault - Bedeutungslosigkeit ne signifierait, dans le chef de Derrida, qu'absence de vouloir-dire, qu'indication et non, comme le lecteur des Recherches logiques pourrait s'y attendre, agrammaticalité : «l'absence d'objet (Gegenstandslosigkeit) n'est donc pas l'absence de vouloir-dire (Bedeutungslosigkeit) ${ }^{1}$. Peut-être faut-il formuler l'hypothèse suivante : fort d'avoir élevé la Gegenstandslosigkeit en structure essentielle $\mathrm{du}$ vouloir-dire, Derrida s'autoriserait à comprendre sous le terme de Bedeutungslosigkeit, l'absence de vouloir-dire. Autrement dit, les Recherches logiques - et plus particulièrement les «distinctions essentielles» de la première Recherche - ne lui fourniraient-elles pas le contexte propice à une telle interprétation traductive du terme Bedeutung ?

En dépit de ces deux hypothèses, le fait que Husserl parle d'une Bedeutungslosigkeit à l'endroit où il sépare le contresens (Widersinnigkeit) et l'agrammaticalité, est un geste tout à fait salutaire pour la téléologie de toute l'interprétation derridienne de la phénoménologie. Cela permet à Derrida de ramener l'agrammaticalité du côté de l'indication et, par là, de réduire cette agrammaticalité. En effet, puisque l'indication ne saurait signifier une Sinnlosigkeit, il est toujours loisible à une indication (Anzeichen ou Bedeuntunglosigkeit) de pouvoir, dans un contexte adapté, avoir un sens plus ou moins déterminé. Et l'indice aura toujours de façon interprétative un sens (Sinn), sinon l'expression occasionnelle je ne s'apparenterait pas à « un indice universellement efficient $»^{2}$. Il peut bien rester bedeutungslos, il n'en demeure pas moins doué d'un certain sens (sinnvoll) mais d'un sens (Sinn) jamais définitivement déterminable, toujours soumis à l'interprétation (Deutung) dans l'horizon indélimitable de contextes insaturables.

Sans ces acquis, Derrida aurait sans doute eu davantage de difficultés à formuler ses notion de " greffe citationnelle », d' « itérabilité »- mobilisées dans la conférence consacrée à la théorie des actes de langage de John Austin - avec lesquels Derrida entend dépasser la conception exclusivement épistémique de l'agrammaticalité husserlienne. Rien n'interdit à une proposition agrammaticale de fonctionner en tant qu'expression catégorématique

${ }^{1}$ Ibid., p. 107.

${ }^{2}$ Ibid. 
significative. L'absence de cette interdiction tient à la structure essentielle du vouloir-dire qu'est la Gegenstandslosigkeit :

C'est sur cette possibilité que je voudrais insister : possibilité de prélèvement et de greffe citationnelle qui appartient à la structure de toute marque, parlée ou écrite, et qui constitue toute marque en écriture avant même et en dehors de tout horizon de communication sémio-linguistique ; en écriture, c'est-àdire en possibilité de fonctionnement coupé, en un certain point, de son vouloir-dire " originel» et de son appartenance à un contexte saturable et contraignant ${ }^{1}$.

Par là, tout type de signe doit pouvoir signifier en dehors de son contexte initial d'énonciation. Si ce n'est pas le cas, la marque signifiante se verrait exposée à l'événementialité empirique et mettrait en danger son idéalité formelle. En dernière instance, Derrida refuse la distinction entre indice et expression, à moins d'accepter le mutisme total de la conscience enfermée dans son soliloque - encore que le chapitre 5 ait montré que l'immédiateté du vécu ne pouvait malgré tout se passer de la médiation rétentionnelle puisque cette distinction mettrait en péril la structure même du signe.

Affirmer la Sinnlosigkeit d'une expression c'est la faire, à suivre l'interprétation derridienne, irrémédiablement glisser du côté de l'empiricité, de la sensibilité brute, c'est vouloir la nier dans son être même de signe. En effet, comme nous le relevions déjà ci-dessus, une fois la distinction entre Sinn et Bedeutung effectuée, seul le chaos des esquisses (Abschattungen), des contenus inarticulés par un sens d'appréhension (Auffassungssinn) peut être dit sinnlos. La Sinnlosigkeit ne s'appliquerait qu'à l'hypothèse - émise au paragraphe 49 des Idées $I$ - de l'impossibilité de faire l'expérience du monde. Par conséquent, aucun type de signe - ni l'indice, ni l'expression ne se trouve concerné par la Sinnlosigkeit; seule la Beteudunglosigkeit inquiète l'espace langagier. Du moins, ce sont les conclusions ultimes que l'on peut tirer d'une lecture de la grammaire pure au prisme de la distinction entre Sinn et Bedeutung.

$\mathrm{Au}$ terme de notre étude, nous espérons avoir pu montrer en quoi la critique de la distinction husserlienne entre l'indice et l'expression repose, chez Derrida, sur l'invalidation d'une différenciation secondaire, celle entre l'absence d'objet donné à l'intuition (Gegenstandslosigkeit) et l'agrammaticalité (Bedeutungslosigkeit). Toutefois, avons-nous fait remarqué qu'une

${ }^{1}$ J. Derrida, «Signature événement contexte », op. cit., p. 381. Malheureusement, Derrida ne donne aucun exemple convaincant d'un énoncé agrammatical devenant, par l'intermédiaire d'un contexte, un énoncé doué d'une signification unitaire. 
telle invalidation devait passer par l'élaboration d'une distinction, inhérente à la lecture des Recherches logiques proposée par Derrida, entre Bedeutungslosigkeit et Sinnlosigkeit. Ainsi, nous avons vu que contrairement au texte des Recherches, ces deux termes ne s'avèrent pas, aux yeux de Derrida, synonymes. Bedeutungslosigkeit recouvre la sphère linguistique tandis que Sinnlosigkeit la sphère noématique. Bedeutungslosigkeit cesse de définir l'agrammaticalité pour plutôt désigner l'absence de vouloir-dire et, par là, l'indice. Au bout du compte, l'agrammaticalité se trouve ainsi complétement ravalée dans l'indication et ce puisque la Sinnlosigkeit ne concerne plus le sens linguistique d'un énoncé mais le sens noématique que mobilisent les actes et les vécus intentionnels. Au bout de l'analyse, l'agrammaticalité a complètement disparu et ce puisque la Sinnlosigkeit, au sein de La voix et le phénomène, cesse de concerner la grammaire. L'indication acquiert une telle étendue, à la suite de la lecture réalisée par Derrida, qu'elle en vient à chasser l'agrammaticalité et à prendre sa place. Les propositions agrammaticales ne relèvent pas de la Sinnlosigkeit mais uniquement de la Bedeutungslosigkeit, comprise en tant qu'indication. Dans La voix et le phénomène, il n'est plus question d'agrammaticalité mais seulement d'absence de vouloir-dire, des propositions indicatives pouvant recouvrir, au gré de différents contextes, une signification expressive. Et d'une certaine manière, toute expression, une fois qu'elle implique l'hic et nunc d'une subjectivité, se trouve contaminée par l'indication et par là, en quelque sorte, par une forme appauvrie à l'extrême de l'agrammaticalité. En ce sens, Derrida ne parvient à faire émerger son supplément originaire que par l'intermédiaire d'une appropriation particulière de ce quasi hapax, au sein des Recherches logiques, qu'est la Bedeutungslosigkeit. 